\title{
Die ungewöhnliche, aber legale Berichterstattung über die Ostverträge im Bundestag vor vierzig Jahren. Ein Zeitzeuge erinnert sich
}

\author{
Claus Arndt
}

Die Geschäftsordnung des Deutschen Bundestages ist materielles Verfassungsrecht. Sie verfällt aber dennoch wie alle Gesetzesvorlagen, Anträge und anderen Vorgänge jeweils mit Ablauf einer Wahlperiode. Es gehört daher zu den ersten Amtshandlungen eines Bundestages nach der Neuwahl und seiner Konstituierung, sich wieder eine Geschäftsordnung zu geben. Üblicherweise wird dafür der Text aus der vorangegangenen Wahlperiode übernommen, es sei denn, der alte Bundestag hatte schon einige Änderungen vorsorglich beschlossen, die dann natürlich in die Neufassung übernommen werden.

In der Geschäftsordnung ist unter anderem das Verfahren geregelt, in dem Gesetzentwürfe behandelt werden. ${ }^{1}$ Normalerweise erfolgt dies in drei Lesungen. Eine Ausnahme bilden Ratifikationsgesetze für internationale Verträge, die nach Artikel 59 GG der Zustimmung des Bundestages bedürfen. Als die Bundesregierung im Winter 1971/72 den gesetzgebenden Körperschaften den Entwurf von Zustimmungsgesetzen für die Verträge von Moskau und Warschau (im Folgenden „Ostverträge“ genannt) zuleitete, überwies das Plenum des Bundestages die Vorlagen an den Auswärtigen Ausschuss federführend und an weitere Ausschüsse - darunter insbesondere den Rechtsausschuss - zur Mitberatung.

$\mathrm{Zu}$ diesem Zeitpunkt beauftragte die größere Regierungsfraktion, die SPD, den Verfasser dieses Beitrags, der stellvertretender Vorsitzender des Rechtsausschusses war, die Vorlagen binnen längstens eines Vierteljahres abstimmungsreif in den Ausschüssen behandeln zu lassen und dafür zu sorgen, dass dem Bundestag rechtzeitig Drucksachen zugeleitet werden können, die einer solchen Schlussabstimmung zugrunde liegen müssen. Ich war damit eine Art „Generalberichterstatter“. Um die Beratung im federführenden und in den mitberatenden Ausschüssen koordinieren zu können, trat ich in den Auswärtigen Ausschuss als stellvertretendes Mitglied ein. Dies war erforderlich, weil der Auswärtige Ausschuss wie der Verteidigungsausschuss ein so genannter geschlossener Ausschuss ist, zu dessen Sitzungen nur die Mitglieder und ihre Stellvertreter Zutritt haben. In allen übrigen Ausschüssen des Bundestages kann jeder Abgeordnete den Beratungen unmittelbar beiwohnen.

Zunächst verabredete ich mit dem Vorsitzenden des Auswärtigen Ausschusses, Gerhard Schröder (CDU/CSU), dass er die dortigen Beratungen der Ostverträge auf die Erörterung der politischen Bedeutung der Vertragswerke im Rahmen der deutschen und europäischen Politik konzentriere. Die Einzelberatung sollte dann im Rechtsausschuss stattfinden, wo ich als stellvertretender Vorsitzender mit über das Ausschuss-Sekretariat verfügen konnte. Anschließend kam ich mit dem Vorsitzenden des Rechtsausschusses, Carl-Otto Lenz (CDU/ CSU) überein, dass die gesamte Arbeit unseres Ausschusses für die Dauer etwa eines Vierteljahres eingestellt werde. Lediglich besonders eilige Vorlagen sollten noch beraten werden können. ${ }^{2}$ Es ist festzuhalten, dass es sich hierbei um einen ungewöhnlichen Vorgang han-

1 Maßgeblich für diese Untersuchung ist die Geschäftsordnung in der Form der Verkündung vom 22. Mai 1970. Vgl. Bundesgesetzblatt I, S. 628.

2 Eine Ausnahme bildete der Entwurf eines Gesetzes zur Neufassung des Seehandelsrechts im Handelsgesetzbuch. Diese weitgehend unpolitische, aber rechtstechnisch relativ aufwendige Arbeit 
delte, weil die praktische Arbeit eines wichtigen Ausschusses für längere Zeit lahmgelegt werden sollte. Da diese Handhabung aber vom Ausschussvorsitzenden und allen Beteiligten mitgetragen wurde, bedurfte es keiner (zahlenmäßig möglichen) Mehrheitsentscheidung. Auch rechtliche Bedenken bestanden nicht gegen ein solches Vorgehen. Die Geschäftsordnung des Bundestages (GO-BT) sagt zu einer solchen Gestaltung der Beratungen nichts aus und steht ihr auch nicht entgegen. So wurden insbesondere Rechte anderer Organe nicht berührt und vor allen Dingen nicht eingeschränkt. Allerdings zeigt $\$ 127$ GO-BT, dass diese Vorschrift generell durchaus unter bestimmten Voraussetzungen Abweichungen im Einzelfall zulässt, wenngleich ihr Wortlaut und ihre systematische Stellung sicherlich nicht unmittelbar auf die Ausschussberatungen anwendbar ist. Demnach dürfte der Schluss erlaubt sein, dass auch die Ausschussberatungen nicht streng an ein bestimmtes Muster gebunden sind, solange die parlamentarischen Beratungen von größtmöglicher Transparenz gekennzeichnet sind und eine Aufteilung der Beratungsmaterien im Allgemeinen eine Erleichterung der Einzelberatungen darstellt. Wenn also derartige Vereinbarungen mehr Transparenz und Effektivität mit sich bringen, dürften ihnen zumindest rechtliche Bedenken nicht entgegenstehen. Sie sind daher rechtmäßig.

Im Rechtsausschuss wurden nunmehr in Sitzungen, die insgesamt 66 Stunden reiner Beratungszeit in Anspruch nahmen, die Zustimmungsgesetze und die beiden Ostverträge beraten. Dabei gab es keine Kleinigkeit oder Nebensächlichkeit, die nicht ausgiebig und verantwortungsvoll untersucht worden wäre. So hat zum Beispiel der Ausschuss sich eingehend mit Unterschieden der Interpunktion im Moskauer Vertrag befasst. Gleichwohl kam er am Schluss dieses Beratungsteils zu dem Ergebnis, dass formal unterschiedliche Schreibweisen in beiden Vertragstexten völkerrechtlich irrelevant seien. Ohnehin stellte die Schlussformel hinter Artikel 5 fest, dass der deutsche wie der russische Vertragstext gleichermaßen für die Auslegung maßgeblich sind. Im Mittelpunkt der Beratungen stand jedoch zunächst die Frage, ob die beiden Vertragspartner Sowjetunion und Bundesrepublik Deutschland in rechtlich einwandfreier und zweifelsfreier Form die zentralen Ziele ihres Vertragsabschlusses deutlich gemacht hatten, denn Zentrum aller Ostverträge war - nach dem Grundsatz „we agree to disagree“ - das Ziel, dass die Vertragsparteien in Zukunft ihre gegenseitigen Beziehungen in friedlicher Weise regeln wollten und dabei auf Gewalt oder die Androhung von Gewalt verzichten würden. Mit anderen Worten: Nicht geregelt werden sollten Statusfragen und völkerrechtliche Festlegungen über die gegensätzlichen Auffassungen der beteiligten Parteien über die Frage des Fortbestandes des Deutschen Reiches oder die rechtliche Bedeutung der Verwaltung der Gebiete jenseits von Oder und Lausitzer Neiße. Die Sowjetunion und Polen vertraten die Meinung, das Deutsche Reich sei mit der Kapitulation der Deutschen Wehrmacht 1945 untergegangen, und es seien nunmehr auf dem Gebiet dieses nach ihrer Meinung früheren Deutschland zwei neue Staaten (DDR und Bundesrepublik Deutschland) entstanden. Die Berichterstattung im Bundestag ging jedoch davon aus, dass die Kapitulation nur der Wehrmacht keineswegs den Untergang des Deutschen Reiches bedeutete. DDR und Bundesrepublik Deutschland seien infolgedessen nur vorläufige Ordnungen auf dem Gebiet des weiterbestehenden deutschen Staates (gegründet 1867 als Norddeutscher Bund, später aber in seinen Gebietsständen - 1871, 1919 oder später - und in seiner Selbstbezeichnung als „Norddeutscher Bund“ oder „Deutsches Reich“ unverändert

übernahm der Abgeordnete Hugo Hauser (CDU/CSU), der als vorzüglicher Jurist und sehr genauer Richter an einem Amtsgericht bekannt war. 
geblieben). Die Einzelberatung dieser Vertragsziele im Rechtsausschuss ergab, dass es den vertragsschließenden Teilen völkerrechtlich tatsächlich gelungen war, die Vereinbarungen zu schließen, ohne damit die genannten Status- und sonstigen Situationen zu verändern.

Eine besondere Rolle spielten die im Abschlussbericht des Rechtsausschusses aufgeführten Materien, über die der Ausschuss nach 50 Stunden reiner Beratungszeit einzeln abstimmte. Insbesondere gilt dies für die Feststellungen in der UN-Charta, des gesamten „Bahrpapiers“, die Frage, ob der Moskauer Vertrag den Prozess der europäischen Einigung nicht behindere, ob das Selbstbestimmungsrecht des deutschen Volkes einschließlich der Rechte auf die Wiedervereinigung beeinträchtigt werde.

Besondere Beachtung fand ebenfalls die sogenannte Feindstaatenklausel in den Artikeln 93 und 107 der UN-Charta und die Vereinbarkeit des Moskauer Vertrags mit anderen Normen des Völkerrechts. Rolle und Status Berlins und die rechtliche Bedeutung des Briefes zur deutschen Einheit wurden ebenso intensiv beraten wie das Verhältnis des Moskauer Vertrags zur Gesamtheit des Grundgesetzes.

$\mathrm{Zu}$ den zahlreichen weiteren Einzelberatungspunkten gehörte nach einem Einleitungsreferat des Abgeordneten Franz-Lorenz von Thadden (CDU/CSU) unter anderem die Erörterung der Frage, welchen Einfluss die Ostverträge auf das rechtliche Schicksal der autochthonen Minderheiten der Kaschuben und der Masuren hatten.

Nach der Geschäftsordnung des Bundestages hat der federführende Ausschuss am Schluss der Beratung aller beteiligten Ausschüsse einen abschließenden Bericht für das Plenum des Bundestages zu verfassen, in den die Berichte der mitberatenden Ausschüsse einzuarbeiten sind. Abweichend von dieser Regelung wurde jedoch zwischen den beteiligten Ausschüssen vereinbart, dass die Berichte nicht in den Text des Auswärtigen Ausschusses eingearbeitet, sondern in vollem Wortlaut diesem angefügt werden sollten. ${ }^{3}$ Auch gegen diese Handhabung bestehen keine rechtlichen Bedenken, obwohl sie im Gegensatz zur jahrzehntelang geübten Praxis im Bundestag steht, denn die Geschäftsordnung gestattet Abweichungen, und wiederum waren alle Beteiligten damit einverstanden. Darüber hinaus bedarf eine hinsichtlich der Abfassung der Drucksache für die Endabstimmung getroffene Absprache der Ausschüsse aber einer intensiveren Untersuchung, weil sie dem Bundestagsplenum unterbreitet wird, also den Rahmen von bloß zwischen Ausschüssen getroffenen Absprachen überschreitet.

Es wird damit Gegenstand der Kompetenz des Bundespräsidenten zu prüfen, ob der ihm unterbreitete Gesetzentwurf nach den Vorschriften des Grundgesetzes zustande gekommen ist (Art. 82 GG). Ein positiver Ausgang dieser Prüfung ist nur dann möglich, wenn die Form der Abschlussdrucksache rechtlich so zulässig ist, wie die Ausschüsse sie getroffen haben. Für diese Zulässigkeit sprechen zunächst all jene Gründe, die oben bereits für die rechtliche Zulässigkeit der Absprache unter den Ausschüssen über die Verteilung der materiellen Beratungsgegenstände dargelegt wurden. Darüber hinaus sind Rechtsvorschriften, die sich speziell gegen diese Übereinkunft richten, nicht erkennbar. Auch die vereinbarte, von allen Beteiligten gebilligte Form der Abschlussdrucksache dient einer Steigerung der Eckpunkte des zentralen parlamentarischen Handelns, der Transparenz und der Effektivität. Sie erleichtert ebenfalls die wissenschaftliche Durchdringung des Stoffs und die Beurteilung der Bedeutung der Ostverträge für Historiker und Juristen. 
Der Bericht des Rechtsausschusses gliederte sich in zwölf Unterpunkte, die der Berichterstatter verfasst und dem Ausschuss zur Abstimmung vorgelegt hatte. Einer wurde einstimmig verabschiedet, alle übrigen wurden von der Mehrheit aus SPD und FDP beschlossen.

Auf der Basis dieser Parlamentsdrucksachen wurden am 17. Mai 1972 die Verträge mit Mehrheit im Plenum beschlossen. Ein großer Teil der CDU/CSU-Fraktion enthielt sich dabei der Stimme. Das Protokoll dieser Plenarsitzung enthält die größte Zahl Persönlicher Bemerkungen, die in der Geschichte des Bundestages von einzelnen Abgeordneten zu einzelnen Abstimmungen abgegeben wurden. Eine ganze Anzahl von ihnen ist sogar in der internationalen Presse nachgedruckt und kommentiert worden. Meine Bemerkungen als Berichterstatter wurden zum Beispiel von fast der gesamten polnischen Presse im Wortlaut wiedergegeben. Der historische Rang dieser Abstimmung ließ es zudem als geboten erscheinen, die Arbeit des Rechtsausschusses an diesen Verträgen international breiter bekannt zu machen. Dem dienten Vorträge vor auswärtigen Gesellschaften in Wien, Paris, Kopenhagen und Oslo, die zum Teil in den außenpolitischen Zeitschriften der entsprechenden Staaten nachgedruckt wurden. ${ }^{4}$ In der sowjetischen Zeitung „Prawda“ wurde seinerzeit ein Interview mit dem Berichterstatter speziell über die laufende Arbeit des Rechtsausschusses an den Ostverträgen gedruckt. ${ }^{5}$ Und heute, fast genau 40 Jahre nach der Abstimmung im Deutschen Bundestag, gilt es daran zu erinnern, welchen Anteil das Parlament und seine Arbeitsstrukturen am Gelingen dieses für die deutsche Geschichte so bedeutenden Vertragswerks gehabt haben.

4 Vgl. Claus Arndt, Rechtliche und politische Aspekte der deutschen Ostpolitik, in: Österreichische Zeitschrift für Außenpolitik, 14. Jg. (1974), H. 6, S. 395 ff.; ders., Les aspects juridiques et politiques de la „Ostpolitik“ allemande de 1970 à 1976, in: Politique Etrangère, 41. Jg. (1976), H. 3, S. 269 - 279; ders., De politiske og retslige sider af Tysklands „Ostpolitik“ 1970/76, in: Fremtiden, 31. Jg. (1976), H. 4, S. 24 ff.; ders., Legal Problems of the German Eastern Treaties, in: American Journal of International Law, 74. Jg. (1980), H. 1, S. $122-133$.

5 In völliger Übereinstimmung mit den Rechtsnormen, Interview in: Prawda (Moskau) vom 8. März 1972, S. 4.

\title{
Wie sehen Parlamentarier den Einfluss der Medien? Ergebnisse einer Befragung der Bundestagsabgeordneten*
}

\author{
Marco Dohle, Christoph Blank und Gerhard Vowe
}

Wie die Auseinandersetzungen vor und nach Rücktritten von Spitzenpolitikern zeigen, wird die Bedeutung von Medien für den Prozess der politischen Willensbildung und Entscheidungsfindung außerhalb und innerhalb der Wissenschaft intensiv diskutiert. Politik, so eine häufig getätigte Aussage, orientiere sich in starkem und zunehmendem Maße an medialen Zeithorizonten, Selektionsregeln und Rollenvorgaben. ${ }^{1}$ Um diese Orientierung an den Me-

* Wir danken der Redaktion der Zeitschrift für Parlamentsfragen für die wertvollen Hinweise, die wir zu einer ersten Version des vorliegenden Textes erhalten haben. 